University of Windsor

Scholarship at UWindsor

$10-2014$

\title{
The Potential of Quinoline Derivatives for the Treatment of Toxoplasma gondii Infection.
}

Sirinart Ananvoranich

University of Windsor

Follow this and additional works at: https://scholar.uwindsor.ca/chemistrybiochemistrypub

Part of the Biochemistry, Biophysics, and Structural Biology Commons, Chemistry Commons, Medicinal and Pharmaceutical Chemistry Commons, Organic Chemicals Commons, and the Pathogenic Microbiology Commons

\section{Recommended Citation}

Ananvoranich, Sirinart. (2014). The Potential of Quinoline Derivatives for the Treatment of Toxoplasma gondii Infection.. Experimental Parasitology, 145, 135-144.

https://scholar.uwindsor.ca/chemistrybiochemistrypub/79

This Article is brought to you for free and open access by the Department of Chemistry and Biochemistry at Scholarship at UWindsor. It has been accepted for inclusion in Chemistry and Biochemistry Publications by an authorized administrator of Scholarship at UWindsor. For more information, please contact scholarship@uwindsor.ca. 


\title{
The potential of quinoline derivatives for the treatment of Toxoplasma gondii infection
}

\author{
7 Q1 Dema Kadri ${ }^{a}$, Anna K. Crater ${ }^{\mathrm{a}}$, Hoyun Lee ${ }^{\mathrm{b}, \mathrm{c}}$, V. Raja Solomon ${ }^{\mathrm{b}}$, Sirinart Ananvoranich ${ }^{\mathrm{a}, *}$ \\ ${ }^{a}$ Department of Chemistry and Biochemistry, University of Windsor, Windsor, Ontario N9B 3P4, Canada

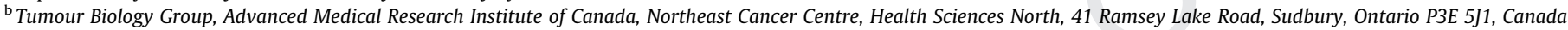 \\ ${ }^{\mathrm{c}}$ Northern Ontario School of Medicine, 935 Ramsey Lake Road, Sudbury, Ontario P3E 2C6, Canada
}

\section{H I G H L I G H T S}

- Quinoline derivatives are effective at inhibiting the growth of Toxoplasma.

- Some quinolones cause the

disappearance of the apicoplast.

- 8-Hydroxyquinoline is very effective

to inhibit the growth of Toxoplasma.
G R A P H I C A L A B S T R A C T

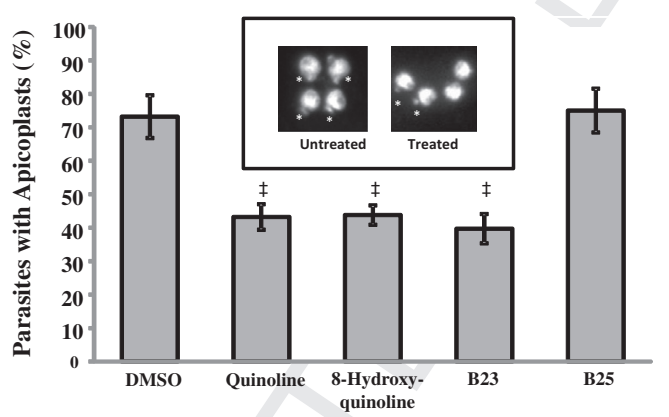

\begin{abstract}
A B S T R A C T
Here we reported our investigation, as part of our drug repositioning effort, on anti-Toxoplasma properties of newly synthesized quinoline compounds. A collection of 4-aminoquinoline and 4-piperazinylquinoline analogs have recently been synthesized for use in cancer chemotherapy. Some analogs were able to outperform chloroquine, a quinoline derivative drug which is commonly used in the treatment of malaria and other parasitic infections. Herein 58 compounds containing one or two quinoline rings were examined for their effectiveness as potential anti-Toxoplasma compounds. Of these 58 compounds, 32 were efficient at inhibiting Toxoplasma growth $\left(\mathrm{IC}_{50}<100 \mu \mathrm{M}\right)$. Five compounds with single and simple quinoline rings exhibited similar cLogP values of $\sim 2$ and $\mathrm{IC}_{50}$ values between 5 and $6 \mu \mathrm{M}$, with one exception of 8-hydroxyquinoline whose $\mathrm{IC}_{50}$ value was $213 \mathrm{nM}$. The addition of one hydroxyl group at position 8 caused a 40-fold increase in the inhibitory effect of quinoline. A significant improvement in anti-Toxoplasma effect among quinoline derivatives was detected in B11, B12, B23, and B24, whose structures carry two quinoline rings, and their resultant cLogP values are $\geqslant 7$. Among these compounds, B23 was the most effective compound with $\mathrm{IC}_{50}$ value of $425 \pm 35 \mathrm{nM}$, and TI value of 4.9 . It was also noted that compounds with at least one quinoline ring, displaying anti-Toxoplasma effects were capable of causing the disappearance of the apicoplast, a plastid-like organelle. When treated with quinoline, 8-hydroxyquinoline or B23, 40-45\% of the parasites lost their apicoplasts. Our findings recapitulate the properties of quinoline derivatives in diminishing apicoplast. This could aid further investigations of anti-parasitic treatments specific to Apicomplexan. More importantly, B12 and B23 which harbor superior anti-cancer properties than chloroquine, have effective anti-Toxoplasma activity. These compounds therefore have significant potential for future development of chemotherapeutic agents for patients suffering from breast cancers and parasitic infection.
\end{abstract}

(c) 2014 Elsevier Inc. All rights reserved.

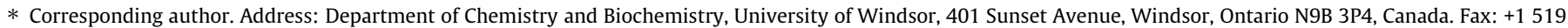
9737098.

E-mail address: anans@uwindsor.ca (S. Ananvoranich). 


\section{Introduction}

Quinoline alkaloids, heterocyclic aromatic organic $\left(\mathrm{C}_{9} \mathrm{H}_{7} \mathrm{~N}\right)$ natural compounds, are known to harbor various medicinal properties and are present in a number of national pharmacopoeias. Their synthetic quinoline counterparts also carry important biological activities. 8-Hydroxyquinoline has growth inhibitory effects against gram positive bacteria and some lower level eukaryotes (Shen et al., 1999), and has been used in the veterinary sciences as an antimicrobial agent for topical treatment (Gershon et al., 1985). The mode of action of 8-hydroxyquinoline is a result of its chemical property as a metal chelator and thought to sequester metals necessary for important enzymes in micro-organisms (Shen et al., 1999). 8-Nitroquinoline has been successfully exploited in the treatment of topical pathogenic parasitic Leishmania infection (Paloque et al., 2012). As early as the 17th century, quinine $[(R)-(6$-Methoxyquinolin-4-yl $)((2 S, 4 S, 8 R)$-8-vinylquinuclidin-2-yl)methanol], an active constituent from the barks of cinchona tree, was used both as a muscle relaxant to halt shivering due to the infection caused by Plasmodium spp. and as an antimalaria agent. Malaria was and remains a serious and sometimes fatal disease. Due to an overwhelming demand for quinine and limited supplies of cinchona barks, the need for readily available and alternative sources of quinine-equivalent compounds gave rise to the discovery of the first synthetic acridine derivative, quinacrine [( $R S)-N^{\prime}$-(6-chloro-2-methoxy-acridin-9-yl)- $N, N$-diethyl-pentane-1,4-diamine], around the time of the First World War (Butler et al., 2010). Soon after the discovery of quinacrine, a more potent quinoline derivative, chloroquine [(RS)- $N^{\prime}$-(7-chloroquinolin-4-yl)$\mathrm{N}, \mathrm{N}$-diethyl-pentane-1,4-diamine], was synthesized (Butler et al., 2010; Solomon et al., 2009). Quinine, quinacrine, and chloroquine are still currently used as anti-malaria agents (Butler et al., 2010). It was hypothesize that these compounds prevent the formation of hemozoin by Plasmodium parasites (Foley and Tilley, 1998). Hemozoin detoxifies free heme intracellularly, as free heme and heme-drug complexes destroy the parasite by creating oxidative stress (Slater, 1992). More detailed structural and functional studies showed that 7-halo substituted compounds are the most active compound. Chloroquine, a 4-aminoquinoline carrying a chlorine moiety at position 7 , is an effective anti-malaria agent currently used in the treatment and prevention of malaria (Butler et al., 2010), and as a reference compound to evaluate the ability of chemicals to inhibit hemozoin formation (Foley and Tilley, 1998). Despite their effectiveness, emerging resistance of Plasmodium spp. has stimulated further drug discovery and development (Butler et al., 2010).

A simple backbone quinoline and 2-methyl-3-carbethoxyquinoline have also been shown to inhibit the growth of Toxoplasma gondii, a Plasmodium-related parasite. When treated with either simple quinoline or 2-methyl-3-carbethoxyquinoline, $52-57 \%$ of Toxoplasma lost their unique organelle called apicoplast (Smith et al., 2007). Similar activity was previously reported with ciprofloxacin, a fluoroquinolone derivative (Fichera and Roos, 1997). It was hypothesized that the crucial pharmacophore conferring this biological activity is the quinoline ring structure (Smith et al., 2007). Interestingly, the presence of an apicoplast is generally used in grouping Plasmodium and Toxoplasma parasites as pathogenic members of the Apicomplexa phylum. The apicoplast is a non-photosynthetic plastid organelle. In Plasmodium and Toxoplasma parasites, the apicoplast is crucial for three essential metabolic functions: the synthesis of heme, type II fatty acids and isoprenoid precursors (Sheiner et al., 2013), and thus has long been considered an ideal target for treating infection caused by Apicomplexan (Fichera and Roos, 1997). Pathogenic Apicomplexan, besides Plasmodium (malaria in humans) and Toxoplasma (toxoplasmosis in humans and animals), are the following. Cryptosporidium can cause cryptosporidiosis whose major symptom in humans is diarrhea (Tzipori and Ward, 2002). Eimeria is the major cause of coccidiosis in agriculturally important animals, including poultry, sheep, and cattle (Shirley and Harvey, 2000). Babesia parasites can cause a hemolytic disease known as babesiosis in humans and occasionally in domestic animals (Vannier and Krause, 2009). Neospora is an important protozoan pathogen in cattle and dogs, while Theileria are important cattle parasites (Katzer et al., 2011).

Drug repositioning can be described as the investigation of new medicinal uses and purposes for previously known and tested drugs (Solomon et al., 2010a, 2010b). Such practices could help overcome high costs and time often associated with the development of new drugs because the known and tested drugs have previously or generally been evaluated or recognized for their pharmacokinetics properties (Chong and Sullivan, 2007). The repositioning studies of chloroquine derivatives (Solomon et al., 2010a, 2010b) have been very valuable in generating a large collection of quinoline derivatives. By combining two important pharmacophores, 4piperazinylquinolones and isatin structure, novel compounds were created with versatile biological effects. For example, B12 and B23 with their hybrid pharmacophore exhibit cancer-specific and are more effective than chloroquine (Solomon et al., 2010b). Due to the presence of multiple structural determinants of the hybrid pharmacophores, these newly generated compounds harbor great potentials to target different sites or molecules, which might result in the possibility to disrupt multiple cellular mechanisms simultaneously and/or to overcome drug resistance (Meunier, 2007).

Here we reported our evaluation for anti-Toxoplasma activity of a wide array of quinoline derivatives, ranging from simple molecules with null or one substitution to multiple substitutions (Tables 1-3). Toxoplasma was chosen as our model organism because (1) it can be easily cultivated in vitro using human fibroblast monolayers (HFF); (2) its doubling time is relatively short ( $\sim 7 \mathrm{~h}$ ) (Seeber and Boothroyd, 1996); (3) the availability of its 2F strain to express beta-galactosidase reporter system offers an accurate and effective growth evaluation (Seeber and Boothroyd, 1996), and (4) protocols for growth inhibition and toxicity assays are well documented (Seeber and Boothroyd, 1996; Jones-Brando et al., 2003). In general, Toxoplasma infection is quite common, affecting a quarter of the world's population, although not all infections lead to lethal toxoplasmosis. Toxoplasma infection can cause very mild or no symptom in healthy individuals, but becomes severe in immunocompromised individuals (Montoya and Rosso, 2005). The infection occurs via ingestion of contaminated food or water. If the infection takes place during pregnancy, the parasite could be transmitted to a fetus and could cause blindness and mental retardation. Current treatments for Toxoplasma infection include pyrimethamine in combination with sulfadiazine and/or clindamycin (Montoya and Rosso, 2005). Pyrimethamine and sulfadiazine inhibit the enzyme dihydrofolate reductase of the tetrahydrofolic acid synthesis, whereas clindamycin inhibits bacterial protein synthesis by inhibiting ribosomal translocation. These current treatments could have severe side effects such as, the inhibition of bone marrow formation and the teratogenic nature of pyrimethamine as a folate inhibitor, precluding it from being used in pregnant women during their first trimester. Sulfadiazine could cause allergic reactions in immunocompromised individuals (Montoya and Rosso, 2005; Peters et al., 2007). Because of these limitations associated with current treatments, it is of great importance to evaluate and find alternative safe treatments. Evaluation of the biological properties of quinoline compounds for their ability to inhibit the growth of Toxoplasma could reap benefits for the ongoing search for effective treatments against the devastating effects of apicomplexan infection. 
Table 1

Simple quinolines.

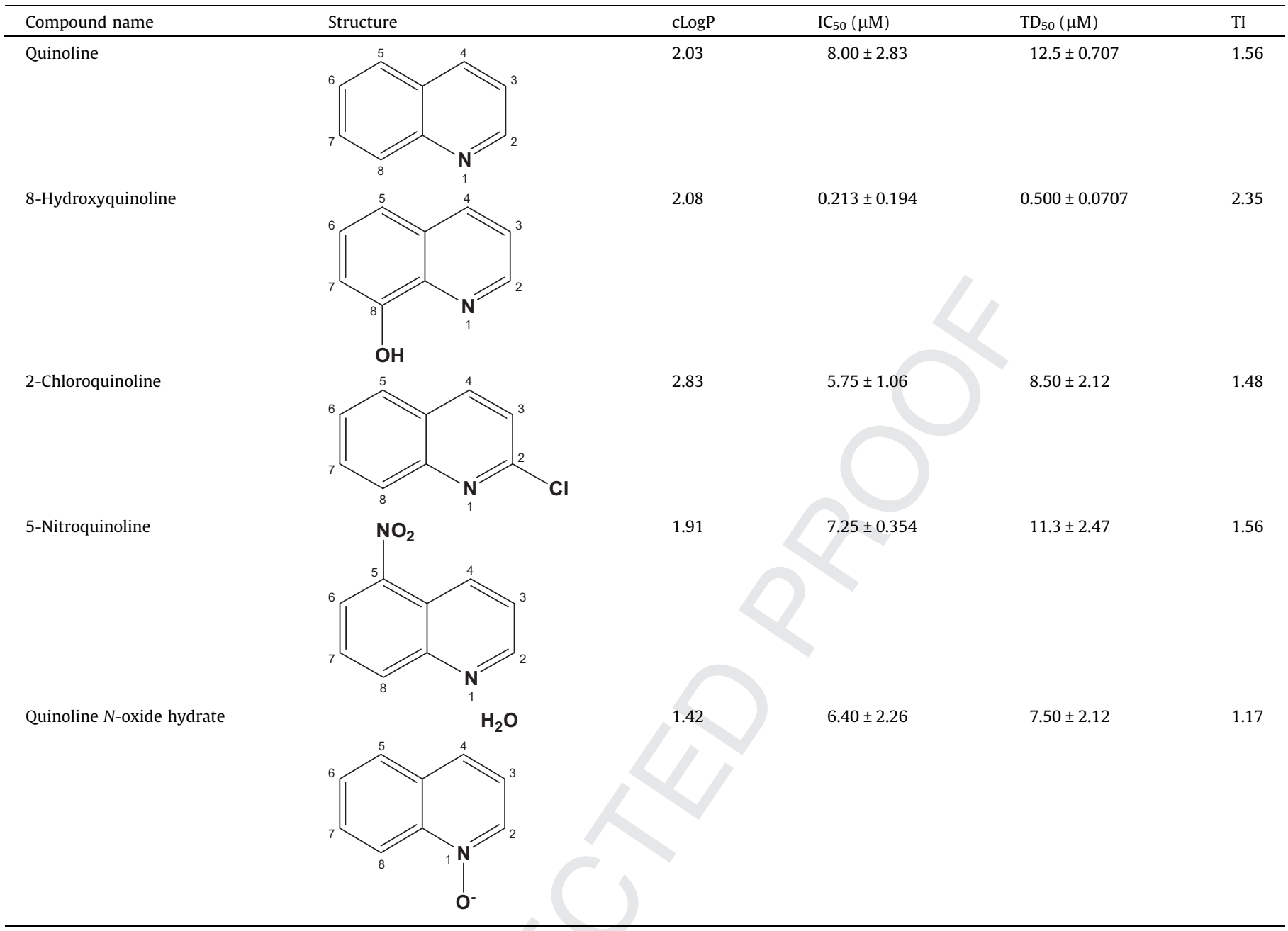

\section{Materials and methods}

\subsection{Chemicals}

Compounds in Table 1 were obtained from Sigma-Aldrich (Canada), while others in Tables 2 and 3 were synthesized and purified according to Solomon et al. (2010a, 2010b). Stock solutions $(10 \mathrm{mM})$ were prepared in DMSO or water, kept at $-20^{\circ} \mathrm{C}$ and diluted to desirable concentrations when needed.

\subsection{Cell and parasite cultures}

HFFs were grown into confluent monolayers for cytotoxicity and parasite's growth inhibition assays using DMEM (Invitrogen, Canada) which was supplemented with $10 \%$ cosmic calf serum (Hyclone, Utah), and 0.5x Antibiotic-Antimycotic (Invitrogen, Canada). Toxoplasma strain $2 \mathrm{~F}$ was obtained from ATCC and proliferated using HFF in MEM containing L-glutamine (Invitrogen, Canada) which was supplemented with $1 \%$ dialyzed fetal bovine serum and 0.5x Antibiotic-Antimycotic and $20 \mu \mathrm{g} / \mathrm{mL}$ gentamycin (Gibco, Canada).

\subsection{Parasite growth inhibition and cytotoxicity assays}

Protocols described by McFadden et al. (1997) and Jones-Brando et al. (2003) were adapted for our study. Briefly
HFF monolayers were grown in 96-well plates and used for evaluation of cellular cytotoxicity of the compounds and their ability to inhibit the growth of Toxoplasma. Immediately prior to the evaluations, their regular culturing media were rinsed twice and replaced with $150 \mu \mathrm{l}$ culturing media [1\% dialyzed fetal bovine serum (Hyclone, Logan, Utah), and 0.5\% Antibiotic-Antimycotic supplemented MEM lacking phenol red, cat \#M3024-10X1L, SigmaAldrich, Canada)]. Tested compounds were added and serially diluted into 11 columns of the plates. Generally the compounds were diluted to achieve concentrations ranging from $10 \mu \mathrm{M}$ to $0.01 \mu \mathrm{M}$. However, for some compounds a higher range $(100 \mu \mathrm{M}$ to $0.1 \mu \mathrm{M})$ was needed. Into the six rows $(6 / 8)$ of the plates, approximately 50 tachyzoites of $2 \mathrm{~F}$ parasites were added and incubated for 4 days at $37{ }^{\circ} \mathrm{C}$ and $5 \% \mathrm{CO}_{2}$. On day 4, chlorophenol Red$\beta$-D-galactoside (CPRG, Sigma-Aldrich, Canada) was added to the wells containing infected monolayers to a final concentration of $100 \mu \mathrm{M}$. On the following day, into two (2/8) rows without the parasite additions, cytotoxicity testing reagent called MTT (3-[4, 5dimethylthiazol-2-yl]-2, 5-diphenyl tetrazolium bromide) (Sigma, Canada) was added to a final concentration of $5 \mathrm{mg} / \mathrm{ml}$. The plates were incubated for $4 \mathrm{~h}$ to allow for tetrazolium salt to form as an indicator of mitochondrial function (Mosmann, 1983). The culture media in the row containing MTT solution was replaced with $100 \mu \mathrm{l}$ of $\mathrm{N}$ - $\mathrm{N}$-dimethylformamide containing $5 \%$ SDS. The products of beta-galactosidase and cytotoxicity assays were purple allowing the absorbance measurement at $560 \mathrm{nM}$ using a Victor3 
Table 2

4-Piperazinylquinoline derived compounds.

\begin{tabular}{|c|c|c|c|c|c|c|c|}
\hline Compound name & Structure & $\mathrm{X}$ & $\mathrm{R}$ & $\operatorname{cLog} \mathrm{P}$ & $\mathrm{IC}_{50}(\mu \mathrm{M})$ & $\mathrm{TD}_{50}(\mu \mathrm{M})$ & TI \\
\hline A2 & & $\mathrm{Cl}$ & 4-Br & 5.03 & $4.25 \pm 0.353$ & $9.97 \pm 2.05$ & 2.35 \\
\hline A3 & & $\mathrm{Cl}$ & $6-\mathrm{Cl}$ & 4.95 & $2.40 \pm 1.41$ & $11.7 \pm 2.88$ & 4.88 \\
\hline A4 & & $\mathrm{Cl}$ & $6-\mathrm{Br}$ & 5.03 & $3.50 \pm 1.414$ & $9.50 \pm 0.707$ & 2.71 \\
\hline A5 & & $\mathrm{Cl}$ & $\mathrm{H}$ & 3.99 & $2.75 \pm 0.354$ & $8.00 \pm 0.707$ & 2.91 \\
\hline A6 & & $\mathrm{CF}_{3}$ & $4-\mathrm{Cl}$ & 5.10 & $4.00 \pm 2.83$ & $7.95 \pm 0.070$ & 1.99 \\
\hline A7 & & $\mathrm{CF}_{3}$ & $4-\mathrm{Br}$ & 5.25 & $7.5 \pm 2.12$ & $14.0 \pm 1.41$ & 1.87 \\
\hline A11 & & $\mathrm{Cl}$ & $4-\mathrm{Cl}$ & 5.18 & $0.895 \pm 0.418$ & $5.00 \pm 0.707$ & 5.59 \\
\hline A12 & & $\mathrm{Cl}$ & $4-\mathrm{Br}$ & 5.33 & $2.45 \pm 0.777$ & $8.00 \pm 1.41$ & 3.27 \\
\hline A14 & & $\mathrm{Cl}$ & $6-\mathrm{Br}$ & 5.33 & $0.800 \pm 0.282$ & $2.80 \pm 0.283$ & 3.50 \\
\hline A15 & & $\mathrm{CF}_{3}$ & $4-\mathrm{Cl}$ & 5.40 & $2.10 \pm 0.141$ & $8.00 \pm 1.414$ & 3.81 \\
\hline A16 & & $\mathrm{CF}_{3}$ & $4-\mathrm{Br}$ & 5.55 & $1.75 \pm 0.354$ & $5.75 \pm 0.353$ & 3.29 \\
\hline A17 & & $\mathrm{CF}_{3}$ & $6-\mathrm{Cl}$ & 5.40 & $1.05 \pm 0.354$ & $4.75 \pm 0.354$ & 4.52 \\
\hline A18 & & $\mathrm{CF}_{3}$ & $6-\mathrm{Br}$ & 5.55 & $0.725 \pm 0.176$ & $3.15 \pm 0.495$ & 4.34 \\
\hline A24 & 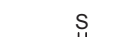 & - & $\mathrm{H}$ & 1.26 & $3.00 \pm 0.707$ & $9.00 \pm 1.41$ & 3.00 \\
\hline
\end{tabular}

microplate reader (Perkin Elmer Life and Analytical Sciences, Woodbridge, ON, Canada). The median inhibitory and cytotoxic doses were used for the calculation, where experimental data were not constrained to $0 \%$ and $100 \%$. The Reed-Muench formula (Reed and Muench, 1938) was applied to obtain the concentration at which $50 \%$ of parasite multiplication $\left(\mathrm{IC}_{50}\right)$ was effectively inhibited, and the concentration at which $50 \%$ of the monolayers exhibited cytotoxic effect $\left(\mathrm{TD}_{50}\right)$. Therapeutic index $(\mathrm{TI})$ was calculated as $\mathrm{TI}=\mathrm{TD}_{50} / \mathrm{IC}_{50}$.

\subsection{Microscopic determination}

HFFs were grown on coverslips and infected with $2 \mathrm{~F}$ for $24 \mathrm{~h}$. The media was replaced with chilled methanol for fixing. Hoechst solution $(10 \mu \mathrm{M})$ was used to stain DNA containing organelles to allow for microscopic determination of apicoplasts. For each test, at least 50 independent fields of view were examined for whether or not the parasites had retained their apicoplasts (Leica fluorescence microscope). Average percentage of the numbers of the parasites with apicoplasts was calculated from each test and for each compound. Three experimental replicates were performed, from which a final average for each compound was obtained. A oneway ANOVA with corrections for multiple comparisons using the Bonferroni method was performed, with alpha set as $0.05 / 5$ to account for different treatment groups (McDonald, 2008). Furthermore, a two tailed student's $t$-test was performed to test the null hypothesis, that there was no statistical difference between the treatment of parasites with DMSO and the tested compounds. The value of alpha, which is associated with the confidence level of the experiments, was set at $0.05(1-0.95)$ for results with $95 \%$ level of confidence. The results were tabulated and used in comparing the effects among the treatments. 


\section{Results and discussion}

\subsection{Toxoplasma growth inhibition and cytotoxicity}

Fifty-eight compounds were divided into three groups; (i) five quinolines with single substituents, (ii) twenty-four analogs of 4-piperazinylquinoline (group A), and (iii) twenty-nine 4-aminoquinoline derivatives (groups B), for which chloroquine was used as reference compound. Notably, DMSO at tested concentrations yielded no adverse effect to HFF and 2F. From the initial round of evaluation, eighteen compounds which did not exhibit a growth inhibition curve were eliminated from the list. Among the remaining forty compounds, eight compounds, which exhibited $\mathrm{IC}_{50}>$ $100 \mu \mathrm{M}$, were deemed less probable for further testing due to their limited potency. The remaining thirty-two compounds were subjected to at least two independent assays of which each assay

Table 3

Chloroquine derived compounds.

\begin{tabular}{lllll}
\hline Compound number & Structure & $\mathrm{CLogP}$ & $\mathrm{IC}_{50}(\mu \mathrm{M})$ & $\mathrm{TD}_{50}(\mu \mathrm{M})$ \\
\hline Chloroquine & $\mathrm{TI}$ & 5.06 & $2.25 \pm 0.354 \quad 9.00 \pm 1.41$ & 4.00 \\
\hline
\end{tabular}

B4<smiles>CS(=O)(=O)OCCCNc1ccnc2cc(C(F)(F)F)ccc12</smiles>

B11<smiles>Clc1ccc2c(NCCCN3CCN(c4ccnc5cc(Cl)ccc45)CC3)ccnc2c1</smiles>

B12<smiles>CCNc1ccnc2cc(Cl)ccc12</smiles><smiles>FC(F)(F)c1ccc2c(N3CCNCC3)ccnc2c1</smiles><smiles>O=C1C(=O)N(CCCNc2ccnc3cc(Cl)ccc23)c2cc(Cl)ccc21</smiles> 
Table 3 (continued)

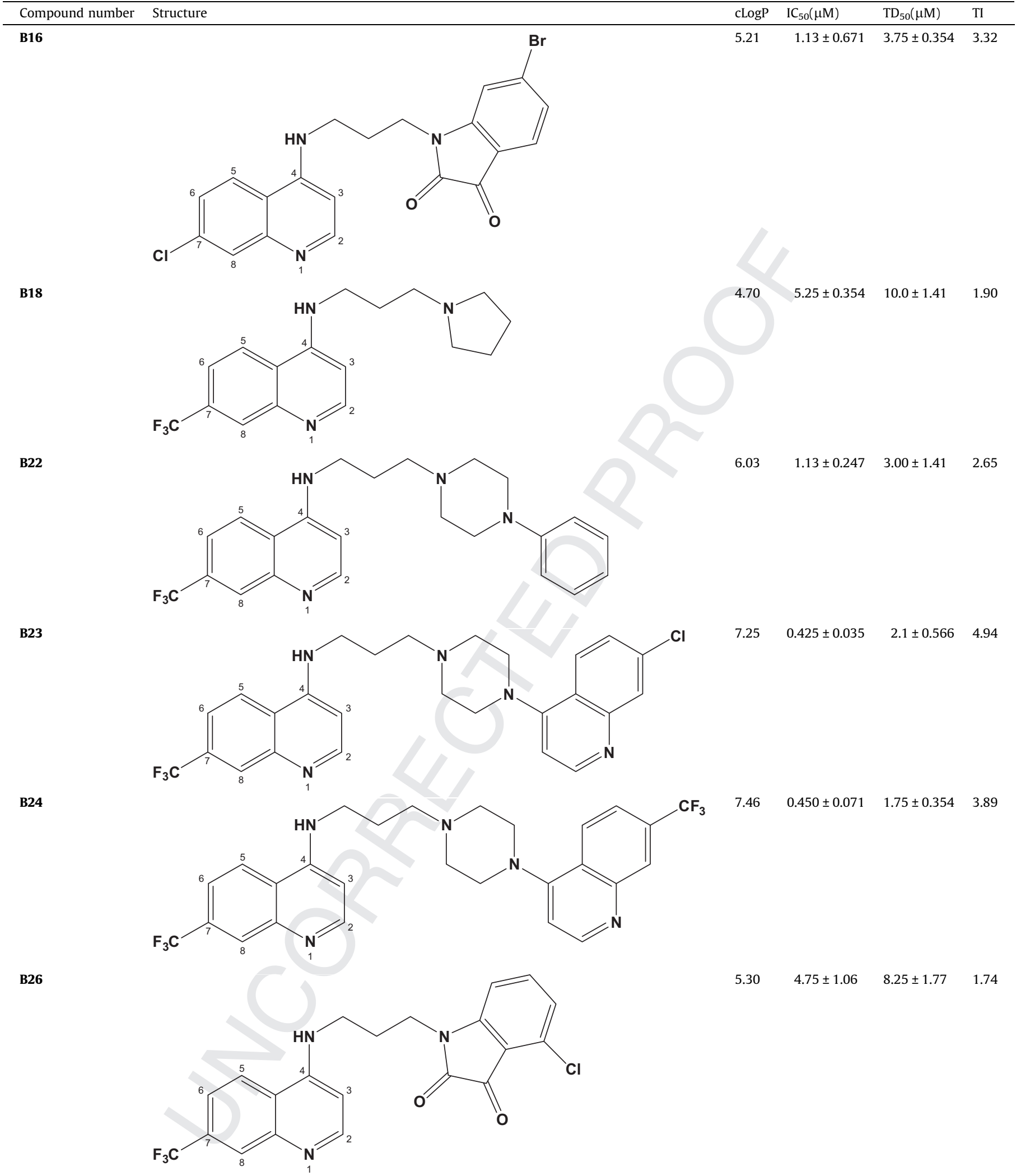


Table 3 (continued)

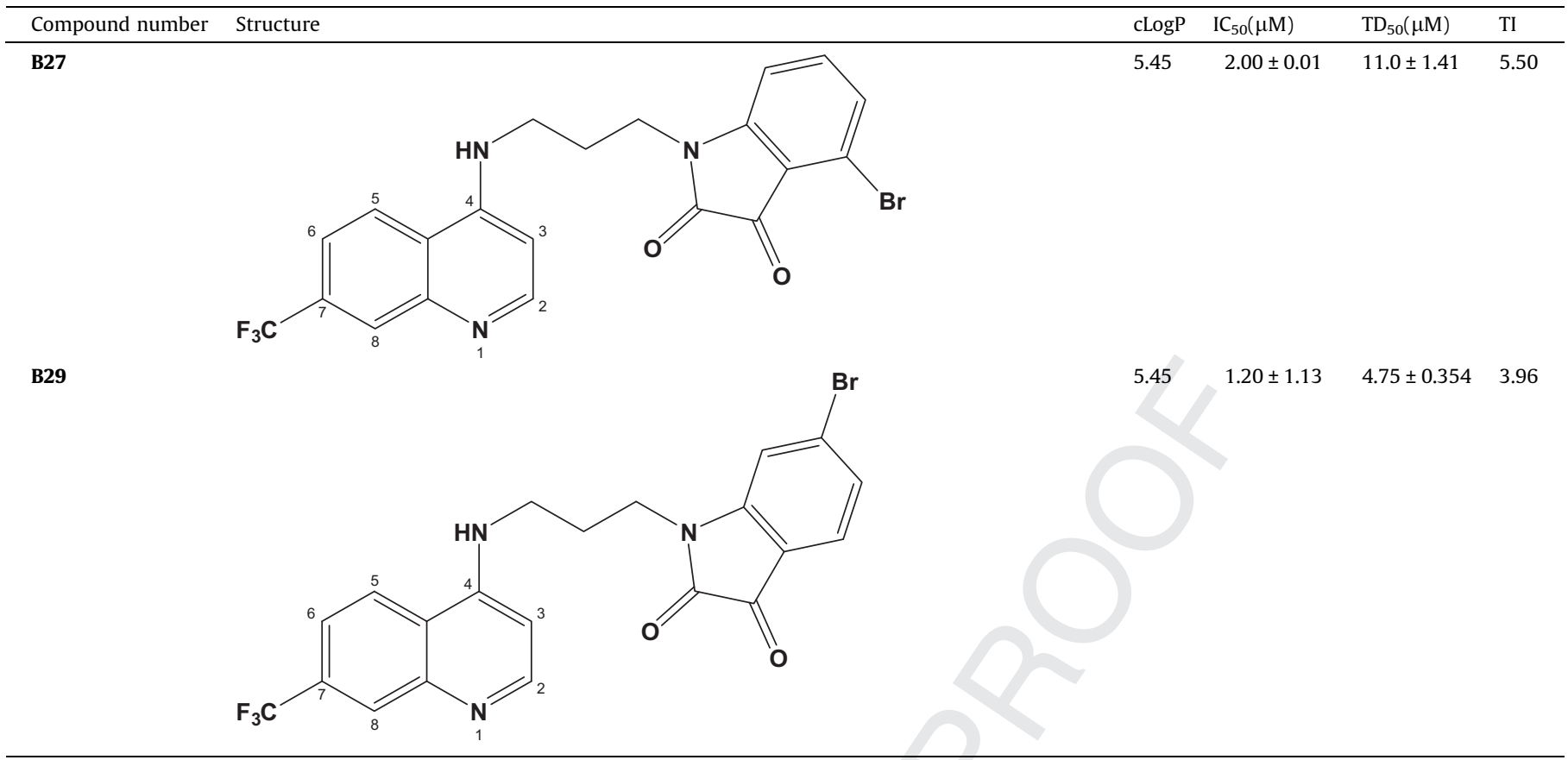

generated at least 8 data points for each test. Their $\mathrm{IC}_{50}$ and $\mathrm{TD}_{50}$ were calculated and analyzed in comparison to their cLogP values.

Simple quinolines (Table 1 ) were tested to verify the hypothesis made by Smith et al. (2007) that the quinoline rings is the active moiety causing growth inhibition. All compounds of this group displayed $\mathrm{IC}_{50}$ at $<10 \mu \mathrm{M}$. Quinoline and 5-nitroquinoline, have cLogP values; 2.03 and 1.91 respectively. The values of cLogP are commonly used to correlate the ability of a compound to cross the cell membranes; a low value indicates that the compound is relatively hydrophilic and less likely to cross the cell membrane. Both compounds have similar cLogP values, which could explain the similarities in their $\mathrm{IC}_{50}$ values at $8.00 \pm 2.83 \mu \mathrm{M}$ and $7.25 \pm 0.354 \mu \mathrm{M}$, and their $\mathrm{TD}_{50}$ values at $12.5 \pm 0.707 \mu \mathrm{M}$ and $11.3 \pm 2.47 \mu \mathrm{M}$, respectively. This suggested that that the 5-nitro substitution did not have a significant effect. 2-Chloroquinoline has a slightly higher cLogP value of 2.83, while showing the $\mathrm{IC}_{50}$ value of $5.75 \pm 1.06 \mu \mathrm{M}$ and $\mathrm{TD}_{50}$ of $8.50 \pm 2.12 \mu \mathrm{M}$. Within this group, quinoline- $N$-oxide hydrate has the lowest $\operatorname{cLog} P$ value at $\sim 1.42$, with the $\mathrm{IC}_{50}$ of $6.40 \pm 2.26 \mu \mathrm{M}$ and $\mathrm{TD}_{50}$ of $7.50 \pm 2.12 \mu \mathrm{M}$, giving this compound the lowest therapeutic index among the series. Interestingly, 8-hydroxyquinoline, with a cLogP value 2.08 , which is close to those of quinoline and 5-nitroquinoline, displayed $\mathrm{IC}_{50}$ of $0.213 \pm 0.194 \mu \mathrm{M}$ and $\mathrm{TD}_{50}$ of $0.500 \pm 0.0707 \mu \mathrm{M}$, resulted in more than a 2 -fold difference in both $\mathrm{IC}_{50}$ and $\mathrm{TD}_{50}$, when compared to the other compounds displaying similar cLogP values. This thus indicated that the 8-subsition of a quinoline ring has the strongest influence on the tested activities. It was not surprising because 8-hydroxyquinoline is known to have antiseptic and antifungal properties against gram positive bacteria (Shen et al., 1999) and used as a topical treatment for skin infections (Gershon et al., 1985). Studies in Plasmodium spp. (Scheibel and Adler, 1982) showed that 8-hydroquinoline could act as a chelating agent, and in Cryptosporidium parvum and related species including T. gondii (Bessoff et al., 2014) also suggested promising uses of 8-hydroquinoline and related quinoline derivatives as anti-parasitic agents. It should be noted that despite a low cLogP value $(\sim 2), 8$ hydroxyquinoline is the most effective, suggesting that it might be up taken by other means than passively traversing the cell membranes.

Compounds of group A (Table 2) were derived from a 4-piperazinylquinoline backbone. These compounds are an example of hybrid pharmacophores, including an isatin ring, a quinoline ring and/or a thiosemcarbazone moiety. These moieties have been reported as important pharmacophores conferring various biological actions and effectiveness in the treatment of cancer (Brockman et al., 1956; Solomon et al., 2009; Hu et al., 2010). The compounds were here presented and compared based on their substituents to the 4-piperazinylquinoline ring system and the nature of the group on the 7-position of the 4-piperazinylquinoline ring. In order to evaluate which of the moieties within these hybrid pharmacophores was eliciting the highest bioactivity, a group of thiosemicarbazone derived compounds with isatin ring systems and not containing a quinoline ring system were tested. The isatin rings were either chloro substituted (A20, A22), bromo substituted (A21, A23), or non-substituted (A24). Of these five compounds, only $\mathbf{A 2 4}$ proved to elicit inhibitory effects with an $\mathrm{IC}_{50}$ of $3.00 \pm 0.707 \mu \mathrm{M}$ and $\mathrm{a} \mathrm{TD}_{50}$ of $9.00 \pm 1.41 \mu \mathrm{M}$. This compound does not contain a quinoline ring and displays an inhibition effect suggesting that the isatin ring system or the thiosemicarbazone, are as active as suggested by (Krivogorsky et al., 2008; Tenório et al., 2005). Compared to simple quinolines described earlier, this group was less effective and showed less potential in Toxoplasma inhibition.

A2-A5 are derived from a 7-chloro-4-piperazinylquinoline ring system with a substituted isatin ring substituted with a 4-bromo (A2), a 6-chloro (A3), a 6-bromo (A4), or a non-substituted isatin ring (A5). All of these compounds proved to have similar abilities at inhibiting Toxoplasma, with the exception of $\mathbf{A 3}$, with an $\mathrm{IC}_{50}$ of $2.40 \pm 1.41 \mu \mathrm{M}$ and $\mathrm{a} \mathrm{TD}_{50}$ of $11.7 \pm 2.88 \mu \mathrm{M}$. Interestingly, $\mathbf{A} 3$ had a similar cLogP value to $\mathbf{A 2}\left(\mathrm{IC}_{50}=4.25 \pm 0.353 \mu \mathrm{M}, \mathrm{TD}_{50}=9.97 \pm\right.$ $2.05 \mu \mathrm{M})$, and $\mathbf{A 4}\left(\mathrm{IC}_{50}=3.50 \pm 1.41 \mu \mathrm{M}, \mathrm{TD}_{50}=9.50 \pm 0.707 \mu \mathrm{M}\right)$. A5, which displayed the lowest cLogP value among the 4 compounds, inhibited the growth of Toxoplasma with an $\mathrm{IC}_{50}$ of $2.75 \pm$ $0.354 \mu \mathrm{M}$ and $\mathrm{a}^{\mathrm{T}} \mathrm{D}_{50}$ of $8.00 \pm 0.707 \mu \mathrm{M}$, and was comparable in activity to A4. A6, A7 were derived from a 7-trifluoromethyl-4- 
piperazinyl ring system substituted with a 4-chloro (A6), 4-bromo (A7) isatin ring. A6 displayed an $\mathrm{IC}_{50}$ value of $4.00 \pm 2.83 \mu \mathrm{M}$ and a $\mathrm{TD}_{50}$ value of $7.95 \pm 0.07 \mu \mathrm{M}$. Within this group, $\mathbf{A} 7$ had relatively high $\mathrm{TD}_{50}$ of $14.0 \pm 1.41 \mu \mathrm{M}$ and $\mathrm{IC}_{50}$ of $7.5 \pm 2.12 \mu \mathrm{M}$. The two bioisoteric groups, with their main chains differing only by the group substituted on the 7-position of the 4-piperazinylquinoline lateral side chain (either $\mathrm{CF}_{3}$ or $\mathrm{Cl}$ group), generally, did not differ much in their biological effects, with the exception of $\mathbf{A} \mathbf{3}$ displaying the greatest TI.

The next analyses were carried out for those compounds which were derived from a 7-chlorosubstituted 4-piperazinylquinoline ring system attached to a substituted isatin-3-thiosemicarbazone ring system to carry either a 4-chloro (A11), 4-bromo (A12), or a 6-bromo (A14) substitution. Among these three compounds, A11 proved to be the most effective displaying an $\mathrm{IC}_{50}$ of $0.895 \pm$ $0.418 \mu \mathrm{M}$ and $\mathrm{a}^{\mathrm{T}} \mathrm{D}_{50}$ of $5.00 \pm 0.707 \mu \mathrm{M}$, with $\mathbf{A 1 4}$ just behind it in terms of effectiveness, the $\mathrm{IC}_{50}$ value of $\mathbf{A 1 4}$ was $0.800 \pm$ $0.282 \mu \mathrm{M}$ and its $\mathrm{TD}_{50}$ value was $2.80 \pm 0.283 \mu \mathrm{M}$, making $\mathbf{A 1 4}$ slightly more cytotoxic to HFF than A11. Of the three compounds, A12 proved to be the least effective with an $\mathrm{IC}_{50}$ of $2.45 \pm 0.777 \mu \mathrm{M}$ and a $\mathrm{TD}_{50}$ value of $8.00 \pm 1.41 \mu \mathrm{M}$. Their bioisoteric counterparts, which contained a 7-trifluoromethyl-4-piperazinylquinoline ring system attached a substituted isatin-3-thiosemicarbazone ring system with either a 4-chloro (A15), 4-bromo (A16), 6-chloro (A17), or 6-bromo (A18) group, were generally similar in their ability to inhibit Toxoplasma, with $\mathbf{A 1 5}$ displaying an $\mathrm{IC}_{50}$ value $2.10 \pm 0.141 \mu \mathrm{M}$ and $a \mathrm{TD}_{50}$ of $8.00 \pm 1.41 \mu \mathrm{M}$, A16 with an $\mathrm{IC}_{50}$ value of $1.75 \pm 0.354 \mu \mathrm{M}$ and a $\mathrm{TD}_{50}$ value of $5.75 \pm 0.353 \mu \mathrm{M}$, with A17 displaying an $\mathrm{IC}_{50}$ value of $1.05 \pm 0.354 \mu \mathrm{M}$ and $\mathrm{a} \mathrm{TD}_{50}$ of $4.75 \pm 0.354 \mu \mathrm{M}$, and $\mathbf{A 1 8}$ displaying an $\mathrm{IC}_{50}$ value of $0.725 \pm$ $0.176 \mu \mathrm{M}$, and $\mathrm{TD}_{50}$ of $3.15 \pm 0.495 \mu \mathrm{M}$. When comparing the two bioisoteric groups, 7-chloro vs. 7-trifluoromethyl on the 4-piperazinyl quinoline ring, those with the 7 -chloro group proved to be slightly more effective. Noted, compounds with a chloro substituted isatin ring were generally the most effective compounds displaying the greatest TI. Taken together, compounds containing the thiosemicarbazone moiety attached to the isatin ring were generally more effective than the compounds containing simply an isatin ring. This could be due the ability of quinoline, isatin, and thiosemicarbazone to inhibit Toxoplasma independently. Additive inhibitory effects on Toxoplasma could result in a more potent pharmacophore. This is similar to the effects observed by Solomon et al. (2010a).

The final set of 29 compounds (group B) was derived from a 4amino quinoline backbone of chloroquine. As a benchmark compound for comparison, chloroquine has a cLogP value of 5.06, $\mathrm{IC}_{50}$ of $2.25 \pm 0.354 \mu \mathrm{M}$ and $\mathrm{TD}_{50}$ of $9.00 \pm 1.41 \mu \mathrm{M}$. Only twelve compounds of this set showed inhibitory effects (Table 3 ) and were compared based on their substituent attached to the 4-aminoquinoline ring system and the nature of the group on the 7-position of the 4-aminoquinoline ring. B11 and B12 were derived from a 7-chloro-4-aminoquinoline side chain substituted with a 4-quinolinyl-piperazinyl chain. B11 has a 4-(7-Chloroquinolin-4-yl)piperazin-1-yl group attached the lateral side chain, while B12 has a 4-(7-trifluoromethylquinolin-4-yl)piperazin-1-yl attached to the lateral side chain, giving these two compounds differ only by one substitution either a $\mathrm{CF}_{3}$ or a $\mathrm{Cl}$ at the 7-position of the 4-quinolinyl-piperazinyl chain. Both do not differ significantly with the $\mathrm{IC}_{50}$ values of $0.875 \pm 0.177 \mu \mathrm{M}$ and $0.750 \pm 0.141 \mu \mathrm{M}$, and their $\mathrm{TD}_{50}$ values of $1.43 \pm 0.513 \mu \mathrm{M}$ and $1.27 \pm 0.550 \mu \mathrm{M}$, with their cLogP values at 7.00 and 7.13 for B11 and B12, respectively. Correspondingly B23 and B24 were derived from the bioisoteric substitution of the 7-chloro group with a 7-trifluoromethyl group on the 4-aminoquinoline ring, with B23 being the bioisoteric counterpart of B11, and B24 of B12. When compared to one another, the activities dis- played by $\mathbf{B 2 3}$ and $\mathbf{B 2 4}$ did not differ significantly in their activities: B23 with an $\mathrm{IC}_{50}$ of $0.425 \pm 0.035 \mu \mathrm{M}$ and $\mathrm{TD}_{50}$ of $2.10 \pm 0.566 \mu \mathrm{M}$, and $\mathbf{B 2 4}$ with an $\mathrm{IC}_{50}$ of $0.450 \pm 0.071 \mu \mathrm{M}$ and a $\mathrm{TD}_{50}$ of $1.75 \pm 0.0 .354 \mu \mathrm{M}$. Also, their cLogP values were similar; B23, 7.25, and B24, 7.46. When compared to their bioisoteric counterparts, no significant difference was observed, however B23 and B24 were active at slightly lower concentrations, which could be due to their greater cLogP values. A substitution of a 4-(7-trifluoromethylquinolin-4-yl)piperazin-1-yl ring system on a 4-aminoquinoline lateral side chain proved to be more effective when there is $\mathrm{a} \mathrm{CF}_{3}$ group at the seventh position of the ring, but not significantly so, as evidenced by the $\mathrm{IC}_{50}$ values of B23 and B24 vs. those of B11 and B12. Notably, the nature of the substituent at the 7-position of the ring, either $\mathrm{Cl}$ or $\mathrm{CF}_{3}$ does not seem to have a significant effect on the parasitic growth inhibition. This is different from what was observed in the proliferation of cancer cells, where a $\mathrm{Cl}$ group substitution at the seventh position of the ring was the more effective agent at inhibiting cancer cell proliferation.

B15 and B16 contain a halogenated isatin ring system on a 7-chloro-4-aminoquinoline lateral side chain. B15 containing a 6-chloroisatin ring, and B16 a 6-bromoisatin ring. In terms of the inhibitory activity, there was no significant difference. B15 with an $\mathrm{IC}_{50} 0.950 \pm 0.071 \mu \mathrm{M}$ and $\mathrm{a} \mathrm{TD}_{50}$ of $5.25 \pm 0.354 \mu \mathrm{M}$, while B16 had an $\mathrm{IC}_{50} 1.13 \pm 0.671 \mu \mathrm{M}$ and $\mathrm{TD}_{50}$ of $3.75 \pm 0.354 \mu \mathrm{M}$. When B26, B27, and B29, derived from a bioisoteric replacement of the 7-chloro group with a 7-trifluoromethyl group on the 4-aminoquinoline ring, were compared, B26 displayed an $\mathrm{IC}_{50}$ of $4.75 \pm$ $1.06 \mu \mathrm{M}$ and $\mathrm{a} \mathrm{TD}_{50} 8.25 \pm 1.77 \mu \mathrm{M}$; B27 an $\mathrm{IC}_{50}$ of $2.00 \pm 0.01 \mu \mathrm{M}$ and $\mathrm{a} \mathrm{TD}_{50}$ of $11.0 \pm 1.41 \mu \mathrm{M}$, and $\mathbf{B 2 9}$ an $\mathrm{IC}_{50}$ of $1.20 \pm 1.13 \mu \mathrm{M}$ and a $\mathrm{TD}_{50} 4.75 \pm 0.354 \mu \mathrm{M}$. The difference in the identity of the group on the isatin substituent, B26 with a 4-Cl, B27 with a 4-Br, and B29 with a 6-Br group yielded slightly different cLogP values: 5.30 for B26, 5.45 for both B27 and B29. Interestingly B27 proved to be more potent and displayed a TI at least two times greater than that of B26 and slightly greater than that of B29.

It should be noted that the compounds containing substituted quinolin-4-yl-4-piperazinyl chains on a 4-aminoquinoline side chain (B11, B12, B23, B24) were more effective in inhibiting Toxoplasma than the compounds containing halo-substituted isatin rings attached to a 4-aminoquinoline lateral side chain (B15, B16, B26, B27, B29). The former group has cLogP values ranging from 7.00 to 7.46 , whereas the latter has cLogP values ranging from 5.06 to 5.45 . The former group contains two quinoline rings, which may have an additive effect in terms of causing inhibition as opposed to a hybrid pharmacophore containing a quinoline and an isatin ring, perhaps further hinting to the potency and efficiency of the quinoline moiety against the growth of Toxoplasma. B4, containing a 7-trifluoromethylaminoquinoline lateral side chain with a methanesulfonate substitution, was more effective than chloroquine with an $\mathrm{IC}_{50}$ value of $1.35 \pm 0.212 \mu \mathrm{M}$, and a $\mathrm{TD}_{50}$ value of $5.67 \pm 1.15 \mu \mathrm{M}$. When compared to the other compounds, which contain at least two ring systems, it was comparable to those compounds containing an isatin ring system substituted on 7-chloro-4-aminoquinoline lateral side chain (B15, B16). The cLogP value of $\mathbf{B} 4$ which is 3.54, this is approximately half of the cLogP values observed in B15 and B16.

When B18, and B22, derived from the 7-trifluoromethylaminoquinoline lateral side chain, were compared for B18 with a pyrrolidinyl group vs. B22 with a phenylpiperazinyl ring substitution. B18 had a relatively high $\mathrm{IC}_{50}$ and $\mathrm{TD}_{50}$ and proved to be less effective than chloroquine. Among chloroquine-derived compounds, B4, B15, B23, and B27 displayed TI values greater than that of chloroquine. B23 was the most potent of this group displaying the lowest $\mathrm{IC}_{50}$ value. Interestingly, B23 was also reported by Solomon et al. (2010a) to be more potent than chloroquine as a cancer 
chemotherapeutic agent. This indicates that this compound may have the potential to be used for multiple purposes. Surprisingly, the most potent compound proved to be 8-hydroxyquinoline among all compounds tested. 4-Aminoquinoline analogs were slightly more potent than 4-piperazinyl quinoline derivatives, particularly B23 and B24 which displayed $\mathrm{IC}_{50}$ ranging from 425 to $585 \mathrm{nM}$.

\subsection{Effect on apicoplast}

Smith et al., (2007) showed that quinoline has an apicoplastdisrupting effect. To evaluate the effect of quinoline derivatives tested here on apicoplast maintenance, quinoline $\left(\mathrm{IC}_{50} 8.0 \pm\right.$ $2.8 \mu \mathrm{M}$ ), 8-hydroxyquinoline ( $\mathrm{IC}_{50} 213 \pm 194 \mathrm{nM}$ ), and B23 $\left(\mathrm{IC}_{50}\right.$ $425 \pm 35 \mathrm{nM}$ ) were selected and tested in comparison to B25 which was an inactive quinoline derivative with $\mathrm{IC}_{50}>100 \mu \mathrm{M}$. To account for various levels of heterogeneity of Toxoplasma cultures, three independent experiments were performed where equal numbers of newly released parasites $\left(\sim 1 \times 10^{6}\right.$ parasites $)$ were used in each experiment. As shown in Fig. 1, due to their growth heterogeneity not all untreated parasites harbored their apicoplasts $(73.2 \pm 6.4 \%)$. The treatments with quinoline, 8 -hydroxyquinoline, or $\mathbf{B 2 3}$ resulted in the loss of apicoplasts, which can be seen by the smaller proportion of parasites maintaining their apicoplasts ( $43.2 \pm 3.8 \%, 43.7 \pm 2.8 \%$, and $39.7 \pm 4.3 \%$, respectively); these data were accounted for $40-45 \%$ loss of apicoplasts, when Toxoplasma were exposed to the drugs. On the other hand B25 did not cause apicoplast loss, in comparison to untreated parasites $(74.9 \pm 6.5 \%)$. The one-way ANOVA analyses yielded $F_{4,10}=36.7$, $P=5.96 \times 10^{-6}$, and $F_{\text {crit }}$ was 3.48. Since the $F$ value generated from ANOVA analyses was greater than $F_{\text {crit }}$ value, it thus indicated significant differences among tested groups. When the Bonferroni correction was applied, the $P$ values obtained was less than the

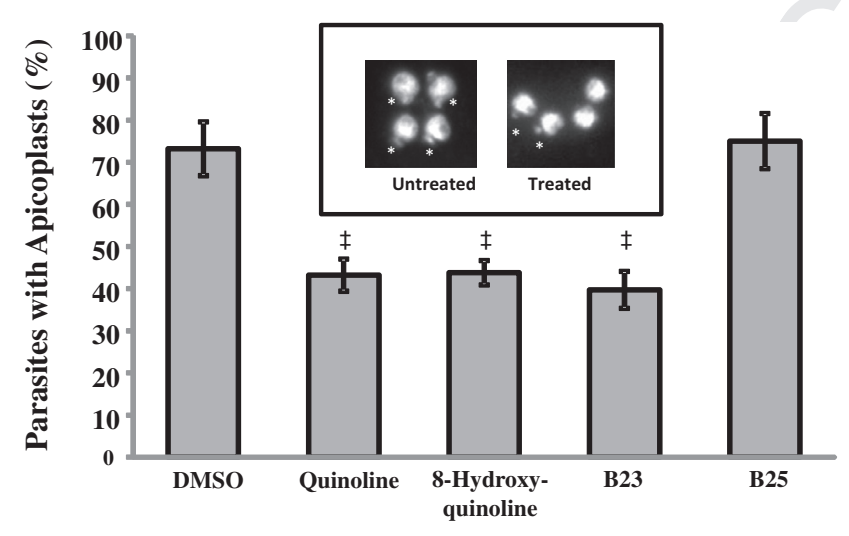

\begin{tabular}{|c|c|c|c|}
\hline & $\begin{array}{c}\text { Average (\%) of } \\
\text { parasites with } \\
\text { apicoplasts }\end{array}$ & Standard deviation & $\boldsymbol{p}$-value \\
\hline DMSO & 73.2 & 6.4 & \\
\hline Quinoline & 43.2 & 3.8 & 0.005 \\
\hline 8-Hydroxyquinoline & 43.7 & 2.8 & 0.007 \\
\hline B23 & 39.7 & 4.3 & 0.003 \\
\hline B25 & 74.9 & 6.5 & 0.754 \\
\hline
\end{tabular}

Fig. 1. Effects of quinoline derivatives on Toxoplasma. Approximately $1 \times 10^{6}$ parasites were used to infect confluent HFF monolayers. At the time of infection, quinoline derivatives were added in the culturing media to obtain the final concentration of 1 or 2 times of $\mathrm{IC}_{50}$, when no cytotoxicity effect was observed. The final concentrations of quinoline were at $8 \mu \mathrm{M} ; 8$-hydroxyquiniline at $426 \mathrm{nM}$; B23, $850 \mathrm{nM}$; B25 $0.8-8 \mu \mathrm{M})$. Twenty-four hours post infection and drug treatments, the parasites were examined as described in Materials and Methods. Double daggers ( $\$)$ indicate $P$-values $<0.05$. Asterisks in the micrograph are for the locations of the apicoplasts. corrected alpha value $(0.01)$, further confirmed the significant difference among the tested groups. To determine which compounds exhibit a statistically significant effect on apicoplast disappearance, a two tailed student's $t$-test was performed, and $P$ values $<0.05$ were obtained. The analysis confirmed the effect of quinoline, 8hydroquinoline and B23 on apicoplast, while B25 showed no effect (Fig. 2).

Although a precise mechanism of disturbance is unknown, our observation is in an agreement with the observations made by Smith et al. (2007). The apicoplast disrupting effect detected for single and two quinoline ring structures suggested stereo-specificity where the single quinoline should be maintained. As seen in the structure of inactive B25, the substitutions differing from that of another quinoline ring did not elicit any biological effect. Further structural elucidation will be required to determine an underlying mechanism by which these compounds target the apicoplast biogenesis, and whether they could be further developed for the treatment of infection caused by apicomplexan. Such an activity was not observed, when pyrimethamine or clindamycin was used in the treatment of infected monolayers (data not shown). Indirectly, it suggested that the effect of quinoline derivatives on apicoplast was specific to the organelle functions, but not due to their general toxicity. However, it is remained to be investigated whether and how quinoline derivatives could affect metabolic functions of apicoplast in the synthesis of heme, type II fatty acids or isoprenoid precursors (Sheiner et al., 2013). In conclusion, B23 has the greatest effect in causing the disappearance of the apicoplast in comparison to the other compounds tested and would be the best candidate for a future study. Undoubtedly, quinoline compounds should be further explored for their effectiveness and selectivity as possible anti-parasitic agents for humans as well as animals.<smiles></smiles>

Quinoline, CLogP 2.03 $(41 \%)$<smiles>Oc1cccc2cccnc12</smiles>

8-Hydroxyquinoline, cLogP 2.08 $(40 \%)$<smiles>FC(F)(F)c1ccc2c(NCCCN3CCN(c4ccnc5cc(Cl)ccc45)CC3)ccnc2c1</smiles>

B23, CLogP 7.25 (45\%)<smiles></smiles>

B25, CLogP $0.82(0 \%)$

Fig. 2. Structural comparisons. The structures of representative compounds exhibiting apicoplast disrupting effects were shown along with their cLogP values and their effect. In parenthesis, the disruption effect was calculated from the proportion of parasite without apicoplast in each test group in comparison to those treated with DMSO solvent. 


\section{Uncited reference}

Solomon and Lee (2009).

\section{Acknowledgments}

This work was supported by funds from the Natural Sciences and Engineering Council of Canada (SA), University of Windsor (SA, DK), and the Northern Ontario Heritage Funds Corporation to HL. We thank Michael Holmes, and Ahmed Cherry and Ross Lepera for their assistance.

\section{References}

Bessoff, K., Spangenberg, T., Foderaro, J.E., Jumani, R.S., Ward, G.E., Huston, C.D., 2014. Identification of Cryptosporidium parvum active chemical series by repurposing the open access malaria box. Antimicrob. Agents Chemother. 58, 2731-2739.

Brockman, R.W., Thomson, J.R., Bell, M.J., Skipper, H.E., 1956. Observations on the antileukemic activity of pyridine-2-carboxaldehyde thiosemicarbazone and thiocarbohydrazone. Cancer Res. 16, 167-170.

Butler, A.R., Khan, S., Ferguson, E., 2010. A brief history of malaria chemotherapy. J. R. Coll. Physicians Edinb. 40, 172-177.

Chong, C.R., Sullivan, D.J., 2007. New uses for old drugs. Nature 448, 645-646.

Fichera, M.E., Roos, D.S., 1997. A plastid organelle as a drug target in apicomplexan parasites. Nature 390, 407-409.

Foley, M., Tilley, L., 1998. Quinoline antimalarials: mechanisms of action and resistance and prospects for new agents. Pharmacol. Ther. 79, 55-87.

Gershon, H., Grefig, A.T., Cady, D.J., 1985. Evidence that the mechanisms of fungitoxicity of 8-quinolinol and its bischelate with copper(II) are different. Can. J. Microbiol. 31, 707-710.

Hu, C., Solomon, V., Cano, P., Lee, H., 2010. A 4-aminoquinoline derivative that markedly sensitizes tumor cell killing by Akt inhibitors with a minimum cytotoxicity to non-cancer cells. Eur. J. Med. Chem. 45, 705-709.

Jones-Brando, L., Torrey, E.F., Yolken, R., 2003. Drugs used in the treatment of schizophrenia and bipolar disorder inhibit the replication of Toxoplasma gondii. Schizophr. Res. 62, 237-244.

Katzer, F., Lizundia, R., Ngugi, D., Blake, D., McKeever, D., 2011. Construction of a genetic map for Theileria parva: identification of hotspots of recombination. Int. J. Parasitol. 41, 669-675.

Krivogorsky, B., Grundt, P., Yolken, R., Jones-Brando, L., 2008. Inhibition of Toxoplasma gondii by indirubin and tryptanthrin analogs. Antimicrob. Agents Chemother. 52, 4466-4469.

McDonald, J.H., 2008. Handbook of Biological Statistics. Sparky House Publishing, Baltimore, Maryland, USA, pp. 115-117.

McFadden, D.C., Seeber, F., Boothroyd, J.C., 1997. Use of Toxoplasma gondii expressing beta-galactosidase for colorimetric assessment of drug activity in vitro. Antimicrob. Agents Chemother. 41, 1849-1853.
Meunier, B., 2007. Hybrid molecules with a dual mode of action: dream or reality Acc. Chem. Res. 41, 69-77.

Montoya, J.G., Rosso, F., 2005. Diagnosis and management of toxoplasmosis. Clin. Perinatol. 32, 705-726.

Paloque, L., Verhaeghe, P., Casanova, M., Castera-Ducros, C., Dumetre, A., Mbatchi, L. Hutter, S., Kraiem-M'rabet, M., Laget, M., Remusat, V., Rault, S., Rathelo, P., Azas, N., Vanelle, P., 2012. Discovery of a new antileishmanial hit in 8-nitroquinoline series. Eur. J. Med. Chem. 54, 75-86.

Peters, P.J., Thigpen, M.C., Parise, M.E., Newman, R.D., 2007. Safety and toxicity of sulfadoxine/pyrimethamine: implications for malaria prevention in pregnancy using intermittent preventive treatment. Drug Saf. 30, 481-501.

Reed, L.J., Muench, H., 1938. A simple method of estimating fifty percent endpoints. Am. J. Hyg. 27, 493-499.

Scheibel, L.W., Adler, A., 1982. Antimalarial activity of selected aromatic chelators III. 8-Hydroxyquinolines (oxines) substituted in positions 5 and 7, and oxines annelated in position 5,6 by an aromatic ring. Mol. Pharmacol. 22, 140-144.

Seeber, F., Boothroyd, J.C., 1996. Escherichia coli beta-galactosidase as an in vitro and in vivo reporter enzyme and stable transfection marker in the intracellula protozoan parasite Toxoplasma gondii. Gene 169, 39-45.

Sheiner, L., Vaidya, A.B., McFadden, G.I., 2013. The metabolic roles of the endosymbiotic organelles of Toxoplasma and Plasmodium spp. Curr. Opin. Microbiol. 16, 452-458.

Shen, A.Y., Chen, C.P., Roffler, S., 1999. A chelating agent possessing cytotoxicity and antimicrobial activity: 7-morpholinomethyl-8-hydroxyquinoline. Life Sci. 64, 813-825.

Shirley, M.W., Harvey, D.A., 2000. A genetic linkage map of the apicomplexan protozoan parasite Eimeria tenella. Genome Res. 10, 1587-1593.

Slater, A.F.G., 1992. Malaria pigment. Exp. Parasitol. 74, 362-365.

Smith, A.T., Livingston, M.R., Mai, A., Filetici, P., Queener, S.F., Sullivan, W.J., 2007. Quinoline derivative MC1626, a putative GCN5 histone acetyltransferase (HAT) inhibitor, exhibits HAT-independent activity against Toxoplasma gondii. Antimicrob. Agents Chemother. 51, 1109-1111.

Solomon, V.R., Lee, H., 2009. Chloroquine and its analogs: a new promise of an old drug for effective and safe cancer therapies. Eur. J. Pharmacol. 625, 220-233.

Solomon, V.R., Hu, C., Lee, H., 2009. Hybrid pharmacophore design and synthesis of isatin-benzothiazole analogs for their anti-breast cancer activity. Bioorg. Med Chem. 17, 7585-7592.

Solomon, V.R., Hu, C.K., Lee, H., 2010a. Design and synthesis of anti-breast cancer agents from 4-piperazinylquinoline: a hybrid pharmacophore approach. Bioorg. Med. Chem. 18, 1563-1572.

Solomon, V.R., Hu, C.K., Lee, H., 2010b. Design and synthesis of chloroquine analogs with anti-breast cancer property. Eur. J. Med. Chem. 45, 3916-3923.

Tenório, R.P., Carvalho, C.S., Pessanha, C.S., de Lima, J.G., de Faria, A.R., et al., 2005 Synthesis of thiosemicarbazone and 4-thiazolidinone derivatives and their in vitro anti-Toxoplasma gondii activity. Bioorg. Med. Chem. Lett. 15, 2575-2578.

Tzipori, S., Ward, H., 2002. Cryptosporidiosis: biology, pathogenesis and disease Microbes Infect. 4, 1047-1058.

Vannier, E., Krause, P.J., 2009. Update on babesiosis. Interdiscip. Perspect. Infect. Dis. 2009, 1-9. 